\title{
RHESSI and Hinode X-ray Observations of a Partially Occulted Solar Flare
}

\author{
Säm Krucker ${ }^{1}$, I.G. Hannah ${ }^{1}$, \& R. P. Lin ${ }^{1,2}$ \\ ${ }^{1}$ Space Sciences Laboratory, University of California, Berkeley, CA 94720-7450, USA \\ ${ }^{2}$ Department of Physics, University of California, Berkeley, CA 94720-7300, USA
}

\begin{abstract}
This letter presents X-ray observations of a partially limb-occulted solar flare taken by the Reuven Ramaty High Energy Solar Spectroscopic Imager (RHESSI) and the X-ray telescope (XRT) onboard Hinode. Thermal emission originates from a simple loop at the western limb that rises slowly $\left(\sim 7 \mathrm{~km} \mathrm{~s}^{-1}\right)$ until the flare peak time. Above $18 \mathrm{keV}$, faint non-thermal emission with a hard/flat spectrum $(\gamma \sim 4)$ and fast time variations (of the order of tens of seconds) is seen that comes from a loop slightly above $(\sim 2000 \mathrm{~km})$ the thermal loop, if compared at the same time. However, the non-thermal loop agrees well in altitude with the thermal flare loop seen later, at the soft X-ray peak time. This is consistent with simple flare models where non-thermal electrons in a flare loop produce thin target hard X-ray emission in the corona as they travel to the loop footpoints. There they lose all their energy and heat chromospheric plasma that fills the loop earlier seen in non-thermal hard X-rays. This suggests that electron acceleration in solar flares occurs in the corona.
\end{abstract}

Subject headings: Sun: flares — Sun: particle emission — Sun: X-rays, gamma rays

\section{Introduction}

The energy stored in magnetic fields in the solar corona is occasionally impulsively released in solar flares. The details when, where, and how this happens are poorly understood (e.g. Miller et al. 1997). The current standard model for solar flares involves release of magnetic energy by the processes of magnetic reconnection of coronal field lines (e.g. Hudson et al. 2004). X-ray and $\gamma$-ray observations show that initially a large fraction of the released energy goes into acceleration of energetic particles. These particles then travel from the corona to the much denser chromosphere where they lose their energy by collisions and heat chromospheric plasma. The heated plasma (typically 10 MK) then fills the flare loops by the process of chromospheric evaporation (e.g. Antonucci et al. 1999). In these models, consecutive reconnection of field lines forms longer and longer loops in time (at least in a two-dimensional model). As the process of evaporation takes time, it is expected that the loops initially containing flare-accelerated particles are later filled with evaporated hot flare 
plasma. This letter presents evidence that flare-accelerated electrons are indeed traveling in loops slightly above the heated flare loops.

Flare-accelerated electrons produce hard X-ray emission by the bremsstrahlung mechanism as they undergo collisions with the ambient plasma. The intensity of the produced hard $\mathrm{X}$-rays fluxes is proportional to the ambient density. Coronal densities are generally too low to significantly slow down flare accelerated electrons and therefore only faint, thin target, hard X-ray emission is produced in the corona. In the chromosphere and below, however, where the density rapidly increases, collisional losses can stop energetic electrons efficiently, producing intense hard X-ray emissions. Hard X-ray imaging observations therefore generally show the most prominent emission from chromospheric footpoints of flare loops, while coronal emission is at least an order of magnitude fainter. Present-day hard X-ray imagers do not provide a large enough dynamic range to regularly observe faint coronal emission in the presence of footpoint emissions. Under favorable conditions, however, coronal emission can be observed as well (e.g. Masuda et al. 1994, Petrosian et al. 2002, Battaglia \& Benz 2006, Krucker et al. 2007). On occasion, coronal densities are high enough to stop most of the flare-accelerated electrons in the corona and coronal emission dominates (Veronig \& Brown 2004). Despite the difficulty of observing non-thermal emissions from the corona, such emissions likely exist in the majority of flares as revealed by statistical studies of partially limb-occulted events (Roy \& Datlowe 1975, McKenzie 1975, Mariska et al. 1996, Tomczak 2001, Krucker \& Lin 2008). In these events the bright hard X-ray footpoints occur behind the solar limb from the observers view and purely coronal emissions can be studied. The observed emission is, as expected, faint and shows a much steeper non-thermal spectrum than what is normally seen from hard X-ray footpoints. With the high spectral resolution (FWHM of $\sim 1 \mathrm{keV}$ ) provided by the Reuven Ramaty High Energy Solar Spectroscopic Imager (RHESSI, Lin et al. 2002) it is possible for the first time to precisely separate thermal from non-thermal emissions and detailed imaging can be done. First results of a statistical survey (Krucker \& Lin 2008) showed that thermal and non-thermal emissions are emitted often spatially close to each other, but can be separated by a few $\operatorname{arcsec}(3000 \mathrm{~km})$. However, large separation with the non-thermal emission occurring $\sim 10 \operatorname{arcsec}(\sim 7000 \mathrm{~km})$ above the thermal loops as seen in the Masuda flare (Masuda et al. 1994) are very rarely seen.

In this paper, a partially limb-occulted flare observed in X-rays by RHESSI and the Xray Telescope (XRT, Golub et al. 2007) onboard Hinode (Kosugi et al. 2007) is presented. The relatively simple flare geometry of a single loop together with the rather flat/hard

photon spectrum allows us to simultaneously image coronal X-ray emission in the thermal and non-thermal range and the test the standard flare model.

\section{Observations and data analysis}

By the time of the observations (November 2006), radiation damage to the RHESSI germanium detectors has started to decrease the effective area and affect the spectral resolution of the instrument. However, not all detectors are influenced in the same way and by using a subset of detectors the effect of radiation damage can be minimized. For imaging, detectors 4, 6, 8, and 9 were used, while detectors 1, 4, and 6 provide the best spectral 
resolution. Furthermore, X-ray observations from XRT are used to study the thermal flare loops. During the flare, XRT was observing with five different filters ('Al-mesh', 'Al-med', 'C-poly', 'Be-thin', 'Be-thick') with a cadence of $150 \mathrm{~s}$. Images taken with the 'Be-thick' filter provide the closest match to the RHESSI observations ( $>3 \mathrm{keV}$ ) as its effective area is mostly around $\sim 2 \mathrm{keV}$ with a FWHM of $\sim 1 \mathrm{keV}$. Hence, both RHESSI and XRT 'Be-thick' data are mostly sensitive to $\geq 10 \mathrm{MK}$ plasma.

\subsection{X-ray time profiles and spectra}

In November 2006, active region AR10923 produced a series of more than 200 rather small flares (mostly below GOES C class). Compared to other events of similar size (Krucker et al. 2002, Christe et al. 2008, Hannah et al. 2008a), these events show non-thermal emissions with extremely hard spectra with power law indices down to $\sim 2$ (Hannah et al. 2008b). The intense non-thermal emission relative to thermal emission makes these events uniquely suited to study bremsstrahlung emissions from flare-accelerated electrons down to energies below $10 \mathrm{keV}$ that otherwise would be hidden in the thermal emission. When AR10923 rotated behind the solar limb, several more similarly sized events occurred. Here we report on the largest (GOES B4) of these partially occulted flares occurring on November 21 around 09:50UT that provides best counting statistics. Similar events, although smaller, occurred on the same day around 00:20UT, 08:30UT, 16:20UT, and 19:30UT. Extrapolating the observed flare locations within AR10923 from times when it was on-disk to the time of the flare presented here suggests a flare location of $7 \pm 2$ arcsec behind the limb (i.e. the loop footpoints are occulted by $5000 \pm 1500 \mathrm{~km}$ ).

Figure 1 shows X-ray time profiles revealing gradually varying thermal emission in the GOES soft X-ray profiles as well in the RHESSI 5-8 keV count rate. The thermal emission is also visible at higher energies, but there is additionally a second component present with short time variations of tens of seconds that is most prominent during the rise of the thermal emission. The fast time variation component is occasionally seen down to very low energies $(\sim 8 \mathrm{keV})$, most clearly around 09:51:26UT. After the peak in the thermal emission, the hard X-ray flux is decaying suggesting that mostly thermal emission is seen at all energies. Above $18 \mathrm{keV}$, the exponentially decaying emission is too faint to be detected above the background. However, earlier on, emission up to $30 \mathrm{keV}$ is observed, confirming that there are two different components in the coronal hard X-ray emission: thermal emission with a gradual time profile, and a rapidly varying component seen at higher energy that is most prominent during the rise phase of the thermal emission. Statistical studies show that these two components are observed in the majority of partially occulted flares (Krucker \& Lin 2008).

Despite the faintness of the fast time variation component, its spectrum above $\sim 18 \mathrm{keV}$ can be fitted with a power law with indices of $\sim 4$ (Figure 2). Statistical results by Krucker \& Lin 2008 showed coronal hard X-ray spectral indices typically around 5.5 with only a few cases with indices as low is observed for this flare. The temporal evolution of the spectrum is soft-hard-soft as observed in the majority of flares (Grigis \& Benz 2004). At lower energies, thermal emission dominates the X-ray spectrum with peak temperatures of $22 \mathrm{MK}(09: 51 \mathrm{UT})$ 
and a maximum emission measure of $2 \cdot 10^{47} \mathrm{~cm}^{-3}$ (09:53UT).

\subsection{X-ray imaging}

Thermal emission in X-rays show a simple flare loop at the western limb without enhanced emissions seen on the disk as expected from a partially occulted flare (Figure 3, middle and right). The thermal RHESSI and XRT 'Be-thick' images show similar source shapes. At this early stage of the Hinode mission, the XRT software does not yet provide accurate pointing information, and the XRT images needed to be shifted by 7 arcsec to the north and 9 arcsec to the west to match the location of the flare loop seen in the RHESSI images. During the impulsive phase of the flare, the flare loop is seen to slowly rise in altitude with a speed of roughly $7 \mathrm{~km} \mathrm{~s}^{-1}$ (Figure 1, bottom). Although the loop only rises by a total of $2000 \mathrm{~km}$, the upward motion is clearly observed by RHESSI, as well by XRT. The location of the flare loop clearly differs from the much weaker pre-flare emission suggesting that pre-flare emission is not coming from the flaring loop. The other XRT filters unfortunately show saturated emission at the location of the flare loop. Together with the RHESSI spectral results, a rough estimate of the plasma density of the flare loop at the peak time can be derived $\left(\sim 2 \cdot 10^{10} \mathrm{~cm}^{-3}\right)$ assuming that flare loop thickness along the line of sight is the same as the observed diameter of $\sim 4$ arcsec. The thermal energy content (e.g. Hannah

et al. 2008a, for definition) in the flare loop is of the order of $\sim 4 \cdot 10^{28} \mathrm{erg}$. During the impulsive phase, the derived densities are about a factor of two smaller.

The fast time variation component seen above $18 \mathrm{keV}$ is much more difficult to image than the thermal emission as the number of counts are much smaller (cf. Figure 1). To maximize the number of counts, only a single image averaged over the total duration of the $>18 \mathrm{keV}$ emission is obtained using the most straightforward imaging algorithm (CLEAN). Note that the CLEANed image has a resolution of $\sim 15$ arcsec FWHM, less than the observed diameter of the XRT loop of $\sim 4$ arcsec. Nevertheless, the $18-30 \mathrm{keV}$ emission is observed to originate from a coronal loop of similar shape as the thermal loop (Figure 3, middle), but it is emitted at a slightly higher altitude $(\sim 2000 \mathrm{~km})$ than the thermal emission seen at the same time. However, the thermal loop at the soft X-ray peak ( $\sim 5$ min later $)$, occurs at the same altitude as the 18-30 keV source (Figure 3, right, and Figure 1, bottom).

\section{Discussion \& Summary}

This letter presents first results of a combined RHESSI and HINODE/XRT X-ray study of a partially occulted solar flare. Even though that the flare footpoints are occulted by the solar limb, hard X-ray emission is observed above $18 \mathrm{keV}$ with fast time variations during the rise phase of the thermal emission. Furthermore, a rather flat/hard X-ray spectrum with power law indices down to 4 is observed. This suggest that the observed emission above $18 \mathrm{keV}$ is bremsstrahlung of flare-accelerated electrons in the corona. A thermal explanation with temperatures up to $100 \mathrm{MK}$ is possible as well, but rather unlikely. Imaging reveals that the thermal emission and the rapidly varying hard X-ray component originate from 
similarly shaped coronal loops, but the thermal component at a slightly lower altitude, if compared at the same time. Later, during the soft X-ray peak of the flare, the thermal component appears to be located in the same loop that contained the rapidly varying component. This is consistent with simple flare models where non-thermal electrons in a flare loop produce thin target hard X-ray emission in the corona while they travel to the loop footpoints where they lose all their energy and heat chromospheric plasma. Chromospheric evaporation then fills the loop earlier seen in non-thermal hard X-rays and makes it radiate in thermal X-ray emission. Together with the observed loop geometry, the observed time delay between brightening in the non-thermal HXR loops and the thermal loop $(\sim 5 \min )$ gives an evaporation velocity of $\sim 60 \mathrm{~km} \mathrm{~s}^{-1}$. This value is of the same order of magnitude as predicted and observed velocities for so-called 'gentle' chromospheric evaporation (Fisher et al. 1985, Abbett \& Hawley 1999, Brosius \& Phillips 2004, Milligan et al. 2006) where only moderate fluxes of flare-accelerated electrons $\left(\leq 10^{10} \mathrm{erg} \mathrm{cm}^{-2} \mathrm{~s}^{-1}\right)$ exit in the flare loop.

The density in the loop with fast time variation emission is difficult to estimate, but it must be lower than the thermal density in the main flare loop of $\sim 2 \cdot 10^{10} \mathrm{~cm}^{-3}$. Therefore, the column density of the loop with fast time variation is $<<4 \cdot 10^{19} \mathrm{~cm}^{-2}$, and that would only stop electrons with energy much less than $20 \mathrm{keV}$. If the accelerated electrons were trapped in the corona so all their energy was deposited there, the hard X-ray emission could be thick target. From the RHESSI spectral observations, the energy deposition in the thick target assumption would be $\sim 2 \cdot 10^{29} \mathrm{erg}$, enough to rapidly heat the loop with fast time variations to $\sim 30 \mathrm{MK}$ (even for ambient densities up to $10^{10} \mathrm{~cm}^{-3}$ ). High temperature loops like this would be easily visible in hard X-rays, even for lower densities. Therefore, the coronal emission seen above $18 \mathrm{keV}$ is most like thin target emission produced by flare-accelerated electrons. This implies that the spectral index of the hard X-ray emitting electrons is as low as $\sim 3$.

The instantaneous number of electrons, $N_{\text {inst }}$, needed to produce the observed hard X-ray emission in the thin target model is straight forward to derived (Brown 1971), but it depends on the ambient density, $n: N_{\text {inst }} \propto n^{-1}$. The total number of needed electrons to produce the observed emission depends how long electrons stay in the corona, $\tau$, and how long the hard X-ray emission last, $\Delta t$. The condition for thin target emission requires that the column density $N=v \tau n$ is much smaller than $N_{\text {thick }} \equiv v(\tau n)_{\text {thick }}=10^{17} E^{2}$, where $v$ and $E$ are the velocity in $\mathrm{cm} \mathrm{s}^{-1}$ and energy in $\mathrm{keV}$ of the non-thermal electron. Using $(\tau n)_{t h i c k}$, a lower limit of the total energy in energetic electrons then can be written as

$$
E_{N T}^{\text {thin }} \sim N_{\text {inst }}<E>\frac{\Delta t}{\tau} \propto \frac{1}{\tau n}>>\frac{1}{(\tau n)_{\text {thick }}}=2 \cdot 10^{28} \mathrm{erg}
$$

where $\langle\mathrm{E}>$ is the average electron energy. In the thin target approximation, these electrons lose negligible energy in the corona, but lose all their energy in the high density chromospheric footpoints. Compared to the thermal energy of $4 \cdot 10^{28}$ erg derived above, there is enough energy in non-thermal electrons to heat the flare loop. Assuming an area of 4 arcsec diameter for the occulted HXR footpoints, the electron flux at the footpoints become $>>2 \cdot 10^{9} \mathrm{erg}$ $\mathrm{cm}^{-2} \mathrm{~s}^{-1}$. This is only a rough order of magnitude approximation, but it is consistent with the limits for gentle evaporation mentioned above.

This letter presents strong evidence for thin target emission from flare-accelerated elec- 
trons in the corona. This thin target emission from the corona indicates that the flareaccelerated electrons are initially in the corona, implying a coronal acceleration region. Favorable conditions made it possible to image the thin target emission: 1) occultation of footpoints, 2) flat/hard spectrum of accelerated electrons, 3) relatively low thermal emission, and 4) a relatively large flare loop. We are planning to search for more of these events to get statistical results on the existence and location of the thin target hard X-ray emission in solar flares.

The work was supported through NASA contract NAS 5-98033 for RHESSI. Hinode is a Japanese mission developed and launched by ISAS/JAXA, with NAOJ as domestic partner and NASA and STFC (UK) as international partners. It is operated by these agencies in co-operation with ESA and NSC (Norway). We would like to thank the Hinode team for making their data available online.

\section{REFERENCES}

Abbett, W. P., \& Hawley, S. L. 1999, ApJ, 521, 906

Antonucci, E., Alexander, D., Culhane, J. L., de Jager, C., MacNeice, P., Somov, B. V., \& Zarro, D. M. 1999, The many faces of the sun: a summary of the results from NASA's Solar Maximum Mission., 331

Battaglia, M., \& Benz, A. O. 2006, A\&A, 456, 751

Brosius, J. W., \& Phillips, K. J. H. 2004, ApJ, 613, 580

Christe, S., Hannah, I.G., Krucker, S., McTiernan, J., Lin, R.P., 2008, ApJ, submitted

Brown, J. C., 1971, Sol. Phys., 18, 489

Fisher, G. H., Canfield, R. C., \& McClymont, A. N. 1985, ApJ, 289, 414

Golub, L., et al. 2007, Sol. Phys., 243, 63

Grigis, P. C., \& Benz, A. O. 2004, A\&A, 426, 1093

Hannah, I.G., Krucker, S., Christe, S., Hudson, H.S., Lin, R.P., 2008b, APS Conference Series, submitted

Hannah, I.G., Christe, S., Krucker, S., Hurford, G.J., Hudson, H.S., Lin, R.P., 2008a, ApJ, submitted

Hudson, H.S., Fletcher, L., Khan, J.I., Kosugi, T., 2004, D.E. Gary and C.U. Keller (eds.), Solar and Space Weather Radiophysics, 153-178

Hurford, G. J., et al. 2002, Sol. Phys., 210, 61 
Kosugi, T., et al. 2007, Sol. Phys., 243, 3

Krucker, S., Christe, S., Lin, R. P., Hurford, G. J., \& Schwartz, R. A. 2002, Sol. Phys., 210, 445

Krucker, S., White, S. M., \& Lin, R. P. 2007, ApJ, 669, L49

Krucker, S., \& Lin, R.P. 2008, ApJ, 673, in press

Lin, R. P., et al. 2002, Sol. Phys., 210, 3

Mariska, J. T., Sakao, T., \& Bentley, R. D. 1996, ApJ, 459, 815

Masuda, S., Kosugi, T., Hara, H., Tsuneta, S., \& Ogawara, Y. 1994, Nature, 371, 495

McKenzie, D. L. 1975, Sol. Phys., 40, 183

Miller, J. A., et al. 1997, J. Geophys. Res., 102, 14631

Milligan, R. O., Gallagher, P. T., Mathioudakis, M., \& Keenan, F. P. 2006, ApJ, 642, L169

Neupert, W. M. 1968, ApJ, 153, L59

Petrosian, V., Donaghy, T. Q., \& McTiernan, J. M. 2002, ApJ, 569, 459

Roy, J.-R., \& Datlowe, D. W. 1975, Sol. Phys., 40, 165

Schmahl, E. J., Pernak, R. L., Hurford, G. J., Lee, J., \& Bong, S. 2007, Sol. Phys., 240, 241

Tomczak, M. 2001, A\&A, 366, 294

Veronig, A. M., \& Brown, J. C. 2004, ApJ, 603, L117

This preprint was prepared with the AAS IATEX macros v5.2. 


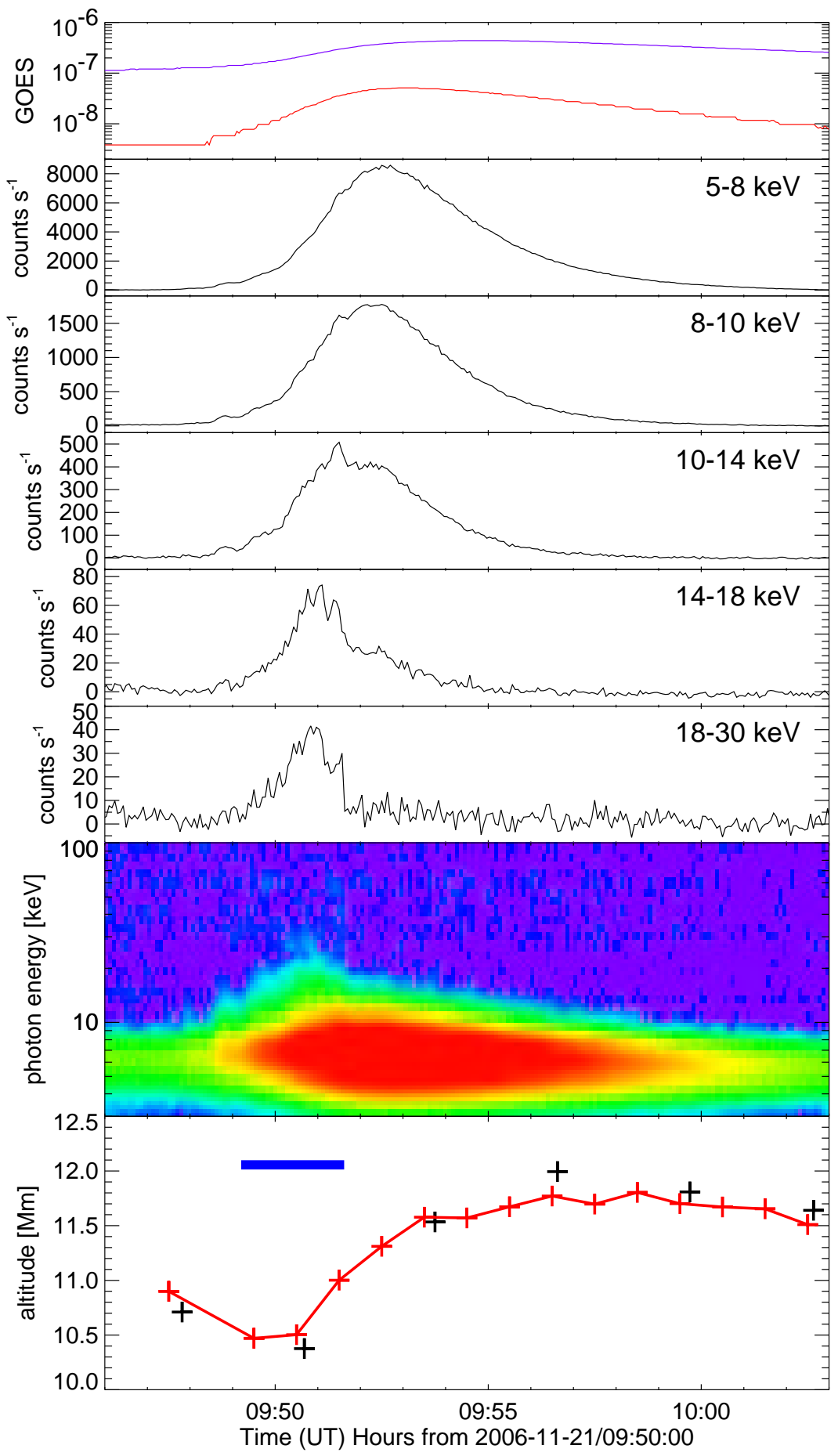

Fig. 1.- X-ray time profiles of the November 21, 2006 event. From top to bottom the panels show GOES soft X-ray flux, RHESSI time profiles at 5 different energy bands, RHESSI spectrogram plot, and the temporal variation of loop-top altitude of the thermal and nonthermal emissions. Altitude of the thermal emissions are shown in red and magenta for XRT and RHESSI, while the altitude of the non-thermal source is given in blue. 


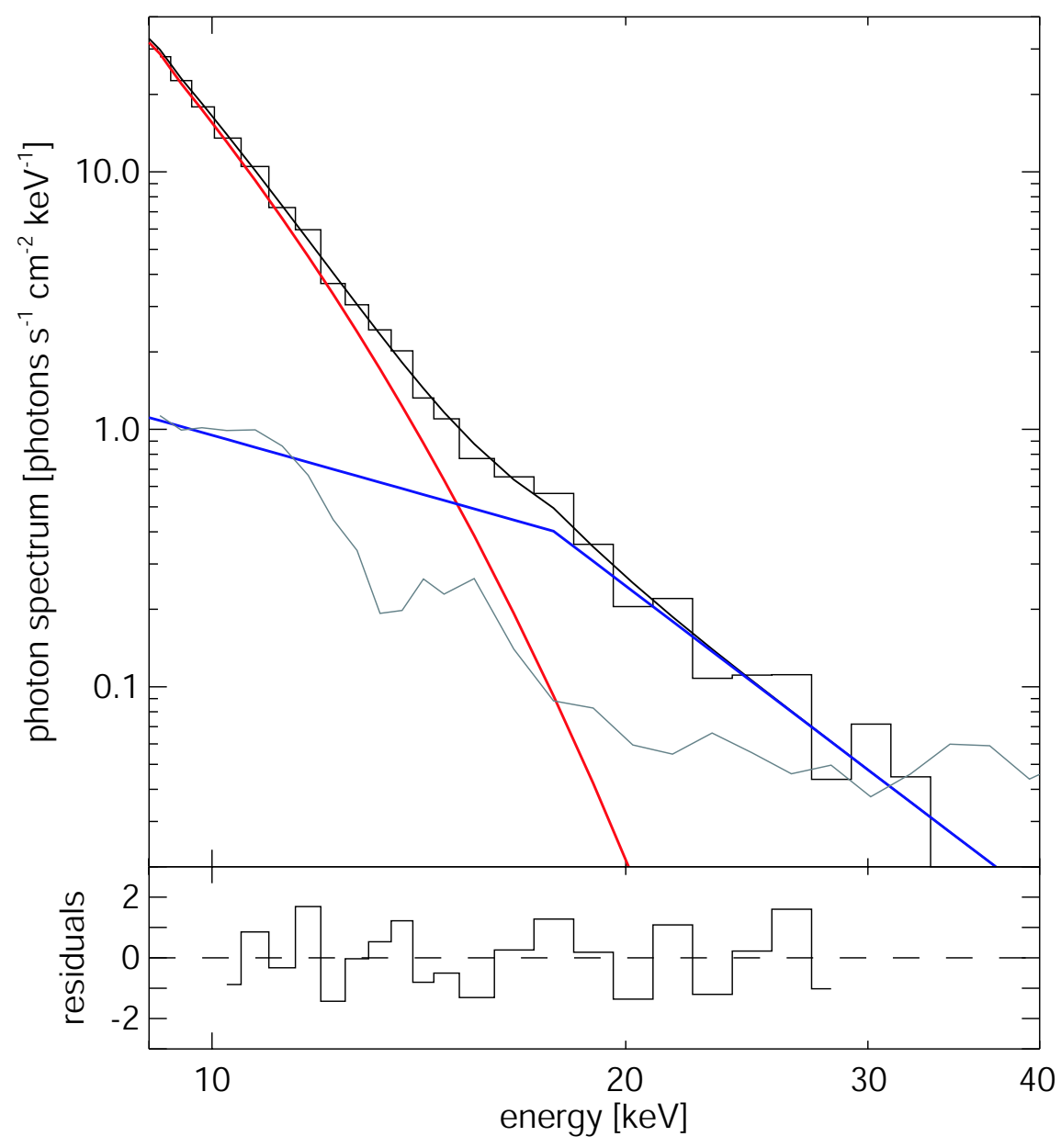

Fig. 2.- Hard X-ray spectrum averaged over $12 \mathrm{~s}$ around 09:50:50UT is shown in black. The observations are fitted with a thermal in red $\left(T=22 \mathrm{MK}, \mathrm{EM}=3 \cdot 10^{46} \mathrm{~cm}^{-3}\right)$ and a broken power law in blue (power law index is 4.1). The gray line represents the background emission, and the panel below shows the normalized residuals of the fit. 

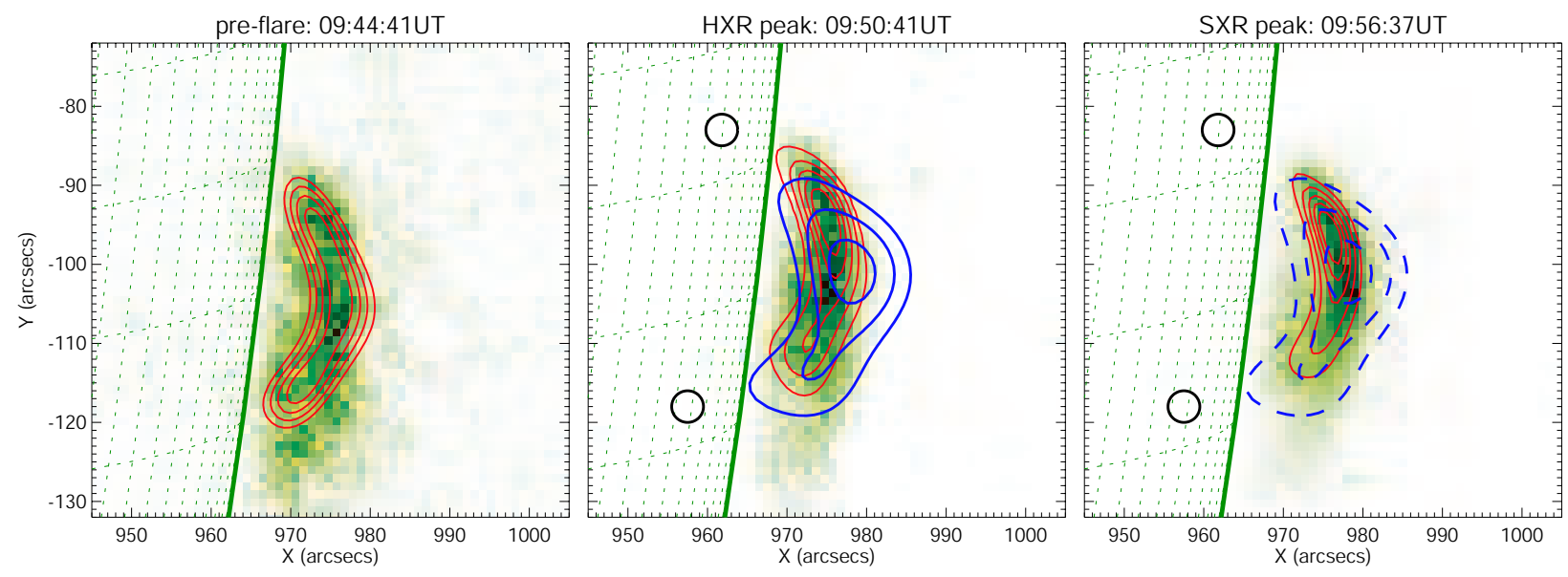

Fig. 3.- X-ray imaging at three different times: (left) pre-flare, (middle) hard X-ray peak, and (right) soft X-ray peak. The shown images are taken by XRT with the 'Be-thick' filter (16 s exposure time). The red contours $(20,35,50,65,80 \%)$ represent 5-8 keV RHESSI images reconstructed with a maximum entropy method using visibilities (Schmahl et al. 2007). The 5-8 keV images of the flare loop are integrated over $32 \mathrm{~s}$, while the pre-flare image is integrated over $120 \mathrm{~s}$. Note that all images are scaled separately, but the pre-flare emission is about 8 times smaller than emission during the soft X-ray peak for XRT $(\sim 2 \mathrm{keV})$, and about 60 times for the $5-8 \mathrm{keV}$ emission. In the two images to the right, the blue contours are 18-30 keV hard X-ray emission averaged over the rise phase of the thermal emission (09:49:12-09:51:44 UT) reconstructed with the CLEAN algorithm (15 arcsec FWHM; 50, $70,90 \%$ contours). The dashed-contours in the right image indicates that the $18-30 \mathrm{keV}$ contours shown are taken earlier, and not during the soft X-ray peak time as the shown thermal emission. The black circle are the expected location of the occulted footpoints of the flare loop. 